\title{
Efficacy of the insect parasitic nematode, Romanomermis iyengari, for malaria vector control in Benin West Africa
}

\author{
Ayaba Z Abagli ${ }^{1}$, Thierv BC Alavo ${ }^{1 *}$, Edward G Platzer $^{2}$ \\ From Challenges in malaria research \\ Basel, Switzerland. 10-12 October 2012
}

\section{Background}

The intensive use of chemical insecticides against mosquitoes has led to the development of widespread insecticide resistance. Control of Anopheles mosquitoes in malariaendemic areas of Sub-Saharan Africa has become increasingly difficult [1]. There is an urgent need for malaria control programs to adopt more integrated mosquito management approaches that include sustainable, nonchemical solutions. In this perspective, insect parasitic nematodes specific to mosquitoes [2,3] may be considered as alternatives, to help reduce reliance on insecticides, and concurrently help insecticide resistance management. The present work has tested the effect of the Mermithid nematode, Romanomermis iyengari, against Anopheles gambiae s.s. Giles in laboratory and field conditions in Benin, West Africa.

\section{Materials and methods}

The nematodes $R$. iyengari were mass produced and the pre-parasitic juvenile (J2) were used in all laboratory and field experiments. Under laboratory conditions, 2 different concentrations of pre-parasitic nematodes ( 5 and $10 \mathrm{~J} 2$ per larvae) were tested against first to third instar (L1, L2 and L3) larvae of $A n$. gambiae. In field, the pre-parasitic nematodes were monthly sprayed into 2 different Anopheles natural breeding sites in Cotonou, south Benin; 3500 and $5000 \mathrm{~J} 2$ per square meter of stagnant water were released, respectively in site 1 and 2 .

\section{Results}

Results indicated that in laboratory, 100\% L1 larvae died within 24 hours post-infection and $100 \%$ of both L 2 and

'Laboratoire d'Entomologie appliquée, Université d'Abomey-Calavi, BP 215 Godomey, Bénin

Full list of author information is available at the end of the article

L3 larvae died within 7 days post-infection, regardless of nematode concentration. In field, Anopheles larval density 5 days post-application decreased from 35 larvae per liter to 4 larvae, and from 17 larvae to 1 , respectively in site 1 and 2. During a whole rainy season in 2011, monthly nematodes spraying resulted in suppression of larval $A n$. gambiae in treated sites.

\section{Conclusions}

The present study indicated that the Mermithid nematode $R$. iyengari is effective for malaria vector control in Benin, West Africa. $R$. iyengari mass production using local materials is easy. Integrating this nematode into An. gambiae management system is therefore possible.

\section{Acknowledgements}

This work has been supported by both Universities of Abomey-Calavi and California, Riverside. The participation of unpaid volunteers for nematodes spraying is highly appreciated.

\section{Author details}

'Laboratoire d'Entomologie appliquée, Université d'Abomey-Calavi, BP 215 Godomey, Bénin. ${ }^{2}$ Department of Nematology, University of California, Riverside, CA 92521-0415, USA.

Published: 15 October 2012

\section{References}

1. Djogbénou L, Pasteur N, Akogbéto M, Weill M, Chandre F: Insecticide resistance in the Anopheles gambiae complex in Benin: a nationwide survey. Medical and Veterinary Entomology 2011, 25:256-267.

2. Platzer EG: Mermithid nematodes. Journal of the American Mosguito Control Association 2007, 23(Sp 2):58-64.

3. Perez-Pacheco R, Rodriguez-Hernandez C, Lara-Reyna J, Montes-Belmont $R$ Ruiz-Vega J: Control of the mosquito Anopheles pseudopunctipennis (Diptera: Culicidae) with Romanomermis iyengari (Nematoda: Mermithidae) in Oaxaca, Mexico. Biological Control 2005, 32(1):137-142.
() Biomed Central

C 2012 Abagli et al; licensee BioMed Central Ltd. This is an Open Access article distributed under the terms of the Creative Commons Attribution License (http://creativecommons.org/licenses/by/2.0), which permits unrestricted use, distribution, and reproduction in any medium, provided the original work is properly cited. 
doi:10.1186/1475-2875-11-S1-P5

Cite this article as: Abagli et al.: Efficacy of the insect parasitic

nematode, Romanomermis iyengari, for malaria vector control in Benin

West Africa. Malaria Journal 2012 11(Suppl 1):P5.

Submit your next manuscript to BioMed Central and take full advantage of:

- Convenient online submission

- Thorough peer review

- No space constraints or color figure charges

- Immediate publication on acceptance

- Inclusion in PubMed, CAS, Scopus and Google Scholar

- Research which is freely available for redistribution

Submit your manuscript at 